Trauma Berufskrankh 2009 • 11[Suppl 1]:91-96 DOI 10.1007/s10039-008-1414-9

Online publiziert: 5 . September 2008

(c) Springer Medizin Verlag 2008

G. Heinrichs ${ }^{1}$ A.-P. Schulz ${ }^{1,2}$. J. Gille ${ }^{1,2} \cdot$ C. Jürgens ${ }^{1,2} \cdot$ A. Paech ${ }^{1,2}$

${ }^{1}$ Sektion für Unfallchirurgie, Klinik für Chirurgie des Stütz- und Bewegungsapparates, Universitätsklinikum-Schleswig-Holstein, Campus Lübeck, Lübeck

${ }^{2}$ Klinik für Unfallchirurgie, BG-Unfallkrankenhaus Hamburg Boberg, Hamburg

\title{
Frakturversorgung an der oberen Extremität
}

\section{Probleme und Problemlösungen}

Die Versorgung von traumatischen Verletzungen der oberen Extremität stellt ein weites Feld dar. Einer Vielzahl verschiedener Verletzungen und Verletzungsmuster auf der einen Seite steht eine große Anzahl unterschiedlicher Versorgungsmöglichkeiten auf der anderen Seite gegenüber. Dabei gilt es, zu beachten, dass es sich oft um komplexe Verletzungen handelt, bei denen es entscheidend ist, die richtige Versorgung für den individuellen Fall zu finden und sich dennoch an standardisierten Abläufen zu orientieren. Im Folgenden beschränken wir uns auf die Darstellung der Versorgung von Frakturen des proximalen Humerus.

\section{Frakturen des proximalen Humerus}

Epidemiologie. Die subkapitale Humerusfraktur macht etwa 5\% aller Extremitätenfrakturen aus [14]. Dabei überwiegt mit 75\% der Anteil an älteren Patienten über 65 Jahren, bei denen meist ein direkter Anprall als Ursache auszumachen ist. Das Verhältnis Männer zu Frauen beträgt bei diesem Patientengut 1:3.

Bei jüngeren Verletzten dagegen stehen Hochrasanztraumen als Mechanismus im Vordergrund, oft handelt es sich um Mehrfachverletzte.

Klassifikation. In der Praxis bewährt hat sich die Einteilung der Frakturen nach Neer (• Abb. 1).

\section{Therapie}

\section{Konservative vs. operative Behandlung}

Wie welche Fraktur zu versorgen ist, wird kontrovers diskutiert. Vergleicht man die Literatur, ist ein Konsens eher bezüglich der konservativen Therapie zu erkennen, mit welcher bei gering dislozierten und stabilen Frakturen in der Regel gute funktionelle Ergebnisse erreicht werden $[8,11$, 13].

Die Indikationsstellung zur operativen Versorgung berücksichtigt viele verschiedene Parameter, die teils patientenabhängig - biologisches Alter, Mobilität und Aktivität des Patienten, Knochenqualität - und teils frakturabhängig - Stabilität der Fraktur, Dislokation, Anzahl der Fragmente - sind.

Bei der operativen Therapie proximaler Humerusfrakturen sind einige Besonderheiten zu beachten, um ein bestmögliches Versorgungsergebnis zu erreichen. Aus anatomischer Sicht handelt es sich dabei um das komplexe Zusammenspiel der Muskeln und Sehnen mit ihren Ansätzen am Tuberculum majus und minus. Um postoperativ eine gute Funktion zu erzielen, ist die korrekte Rekonstruktion der Rotatorenmanschette mit ihren Muskelansätzen zwingend erforderlich. Ein weiterer Faktor ist die fragile Blutversorgung des Humeruskopfes, für welche eine intakte mediale Periostverbindung von entscheidender Bedeutung ist [2].
Abgesehen von den anatomischen Aspekten ist die ausreichende präoperative Diagnostik samt Bildgebung für das Outcome entscheidend. Dabei sollten v. a. die Anzahl der Fragmente und die Stellung der Fraktur bekannt sein, um die richtige Versorgungsform wählen zu können. Die weitere präoperative Diagnostik sollte immer die Beurteilung von Begleitverletzungen umfassen, wobei die Verletzung von Nerven, in diesem Fall insbesondere des N. axillaris, im Vordergrund steht. Bei klinischem Verdacht empfiehlt sich die Durchführung einer neurologischen Untersuchung, ggf. auch einer elektrophysiologischen Validierung des Befunds.

\section{Operation}

\section{Indikationen verschiedener Versorgungsformen}

Folgt man der Literatur, besteht die Indikation für die Osteosynthese von Frakturen des proximalen Oberarms mittels eines proximalen Humerusnagels v. a. in den Neer-Frakturtypen III, IV/3, V/3 sowie relativ bei den Typen IV/V4, IV/3 und VI/4 [10]. Dagegen stellt eine Head-SplitFraktur eine Kontraindikation für die osteosynthetische Versorgung dar. Frakturen mit 4 oder mehr Fragmenten sollten in der Regel eher mit einer Platte oder im Zweifel mit einer Endoprothese versorgt werden $[3,12]$.

\section{Grundlagen der Versorgung}

Bereits die korrekte Lagerung ist ein wichtiger Schritt der erfolgreichen Versorgung, 


\section{Der vermeintlich einfache Fall}

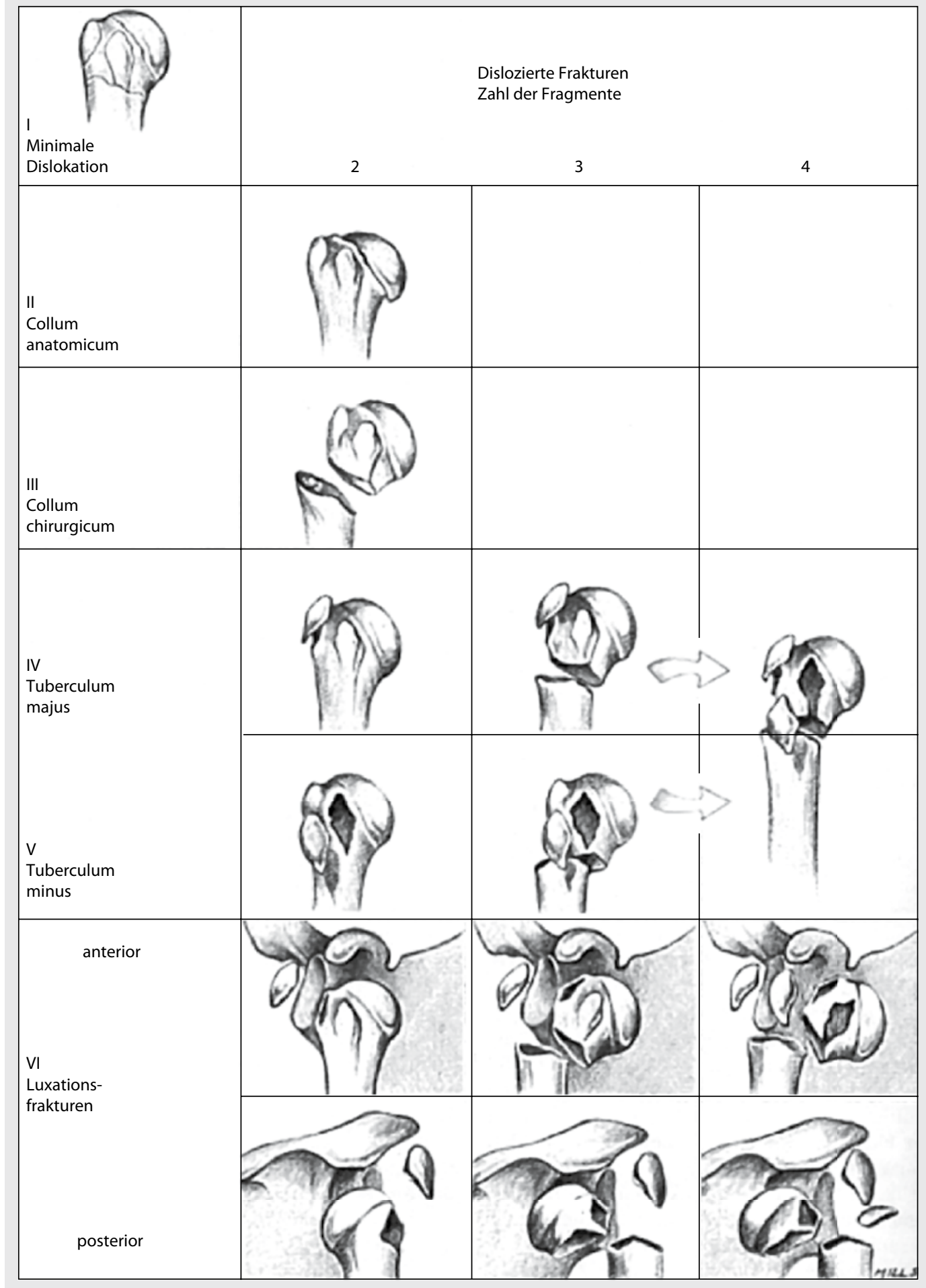

Abb. $1<$ Klassifikation nach Neer

da nur dann eine ausreichend gute Möglichkeit zur intraoperativen Bildwandlerkontrolle in mehreren Ebenen gegeben ist. Die Standardlagerung besteht in einer Beach-Chair-Lagerung auf dem Schultertisch, bei der der Unterarm auf einer Standardarmstütze gelagert wird.

Ein kurzer Deltasplitzugang ermöglicht den Zugang zum Humeruskopf. Er kann ggf. verlängert werden, um eine korrekte Reposition zu ermöglichen, die für das funktionelle Ergebnis entscheidend ist.

Die Nachbehandlungsschemata sind vielfältig und variabel. In der Schulterendoprothetik und bei der Versorgung komplexer Mehrfragmentfrakturen sowie bei osteoporotischem Knochen hat sich un- serer Erfahrung nach das Schema nach Habermeyer [5] bewährt.

\section{Grundlegende Techniken der Versorgungsmöglichkeiten}

Osteosynthese der proximalen Humerusfraktur mittels winkelstabilem Humerusnagel (z. B. PHN, Fa. Stryker). Einer der 
entscheidenden Operationsschritte ist die korrekte Bestimmung des Eintrittspunkts (- Abb. 2). Dieser sollte in der Verlängerung der Humerusschaftachse und medial des Tuberculum majus im Bereich des Kalottenknorpels liegen. Bei zu lateraler Positionierung des Nagels ist eine Dislokation des lateralen (Tuberculum-majus)-Fragments zu befürchten. Bei richtiger Positionierung ist auch bei osteoporotischem Knochen in der Regel im Fräszylinder spongiöser Knochen mit hoher Festigkeit anzutreffen (• Abb. 3).

Der Nagel wird knapp unter dem Knorpelniveau versenkt, der spongiöse Knochen dient als zusätzliches stabilisierendes Element. Eine weitere Stabilisierung wird über die Anordnung der Schrauben erreicht, die in den meisten Fällen über das entsprechende Zielgerät eingebracht werden. Des Weiteren ist darauf zu achten, möglichst alle vorhandenen Verriegelungsoptionen mit Schrauben zu besetzen.

\section{Versorgung mittels einer winkelstabilen} Plattenosteosynthese. Sie spielt zunehmend auch bei komplexen 3- und 4-Fragment-Frakturen eine Rolle. Wichtig für die Stabilität ist die divergierende Lage der Schrauben im Kopfbereich. Gemeinsam mit den winkelstabilen Eigenschaften der Platte ist nur so auch bei osteoporotischem Knochen eine ausreichende Stabilität zu gewährleisten. Dabei werden im Kopfbereich Spongiosaschrauben und im Schaftbereich Kortikalisschrauben verwendet.

Sowohl beim Nagel als auch bei der Plattenosteosynthese sollten die Schrauben im Kopfbereich idealerweise bis unmittelbar an die Gegenkortikalis heranreichen.

Endoprothese. Die Indikationsstellung zur primären Versorgung mit einer Schulterendoprothese wird kontrovers diskutiert. Diese Möglichkeit gewinnt jedoch zunehmend an Bedeutung [3, 12, 16].

Beim posttraumatischen Gelenkersatz werden häufig Hemiprothesen verwendet.

Entscheidend für ein ausreichend gutes funktionelles Ergebnis sind die korrekte Positionierung der Prothese sowie die Wahl der richtigen Kopfgröße, um
Trauma Berufskrankh 2009 · 11[Suppl 1]:91-96 DOI 10.1007/s10039-008-1414-9

(C) Springer Medizin Verlag 2008

\section{G. Heinrichs · A.-P. Schulz · J. Gille · C. Jürgens · A. Paech Frakturversorgung an der oberen Extremität. Probleme und Problemlösungen}

\section{Zusammenfassung}

Die subkapitale Humerusfraktur macht etwa 5\% aller Extremitätenfrakturen aus, 75\% der Betroffenen sind über 65 Jahre alt. Die Frakturen werden nach der Neer-Klassifikation eingeteilt. Bei gering dislozierten und stabilen Frakturen sind durch konservative Behandlung in der Regel gute funktionelle Ergebnisse erzielbar. Die Indikationsstellung zur operativen Versorgung berücksichtigt verschiedenste Parameter, die teils patienten-, teils frakturabhängig sind. Eine ausreichende präoperative Diagnostik samt Bildgebung ist Grundvoraussetzung für ein optimales postoperatives Ergebnis. Die korrekte Rekonstruktion der Rotatorenmanschette ist zwingend erforderlich. Ähnliches gilt hin-

sichtlich der fragilen Blutversorgung des $\mathrm{Hu}$ meruskopfes, für welche eine intakte mediale Periostverbindung entscheidend ist. Bei der Wahl der Versorgungsform - Osteosynthese mittels proximalem Humerusnagel, Plattenosteosynthese oder Endoprothese sollten neben Frakturtyp, -stellung, Zahl der Fragmente usw. auch individuelle Besonderheiten des Patienten selbst sowie dessen Anforderungen berücksichtigt werden, um eine optimale Versorgung zu erreichen.

\section{Schlüsselwörter}

Proximale Humerusfrakturen - Diagnostik . Versorgung $\cdot$ Verfahrenswahl $\cdot$ Praxistipps

\section{Treatment of upper extremity fractures. Problems and problem-solving}

\section{Abstract}

The subcapital humerus fracture accounts for approximately $5 \%$ of all extremity fractures. Of this patient population $75 \%$ are over 65 years. Fractures are classified according to the Neer score. In the case of mildly displaced and stable fractures, good functional results can generally be achieved with conservative therapy. The indication for surgery takes varying parameters -- some fracture-specific, some patient-specific -- into account. Adequate preoperative diagnosis, including imaging, is the basic prerequisite for optimal postoperative results. Correct reconstruction of the rotator cuff is mandatory. The same is true for the fragile blood supply to the hu- meral head, for which an intact medial periosteal connection is crucial. When choosing the method of treatment -- osteosyntheses using a proximal humerus nail, plate osteosynthesis or endoprostheses -- fracture type and status, number of fragments, etc. as well as the individual characteristics of patients and their expectations should all be taken into consideration in order to provide optimal treatment.

\author{
Keywords \\ Proximal humeral fracture · Diagnosis . \\ Therapy · Choice of method P Practical tips
}




\section{Der vermeintlich einfache Fall}

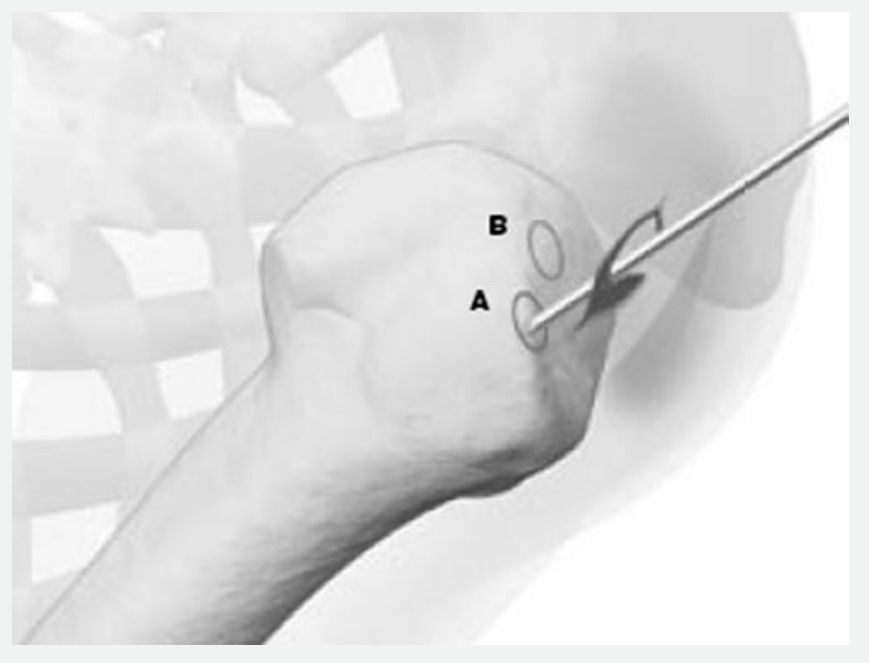

Abb. 24 Korrekter Eintrittspunkt als entscheidende Grundlage für die richtige Positionierung eines Marknagels, Punkt B sollte verwendeter Position entsprechen
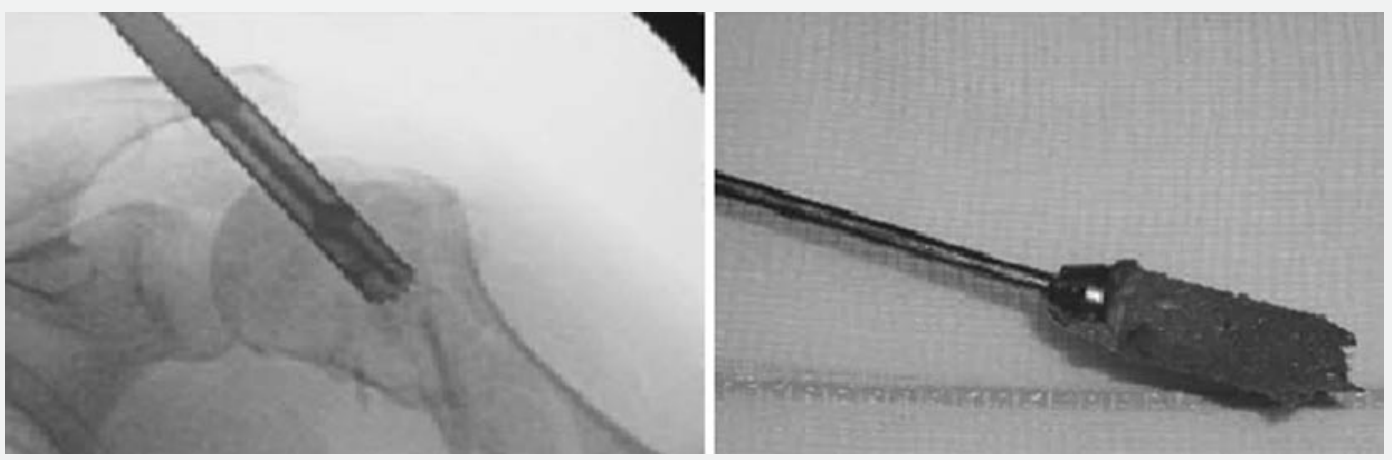

Abb. $3 \varangle$ Fräszylinder mit spongiösem Knochen

ein korrektes Artikulieren mit der Schulterpfanne zu ermöglichen, ohne dabei einerseits eine Luxationstendenz aufzuweisen oder andererseits den Bewegungsumfang unnötig einzuschränken.

\section{Unspezifische und versorgungs- spezifische Komplikationen und Lösungsansätze}

Der erste Schritt zur Vermeidung von Komplikationen muss in der korrekten Indikationsstellung liegen. Dabei ist eine genaue Kenntnis der Fraktur von entscheidender Bedeutung. Um diese zu erhalten, empfiehlt es sich, die präoperative Planung bei komplexeren Frakturen durch ein CT zu ergänzen [9]. Mit Kenntnis der Bilder und unter Berücksichtigung der patientenspezifischen Besonderheiten und Bedürfnisse, aber auch der Compliance des Patienten sollten dann das entsprechende Operationsverfahren bzw. das richtige Implantat gewählt werden.

Unabhängig vom verwendeten Implantat ist die korrekte Reposition der Fragmente ein wiederkehrendes Problem. Über den kurzen Deltasplitzugang ist nicht immer eine ausreichende Sicht zu gewährleisten. Muss der Deltasplit verlängert werden, wird der Verlauf des N. axillaris zum möglichen Problem (• Abb. 4). Um eine korrekte Reposition zu erreichen, sind in bestimmten Fällen die $\mathrm{Zu}$ hilfenahme einer temporären KirschnerDraht-Osteosynthese oder das Anschlingen einzelner Muskelsehnen empfehlenswert [7]. Insbesondere die korrekte Reposition des Kalottenfragments ist entscheidend [6]. Bei subkapitalen Humerusfrakturen ist auf die korrekte Rotation des Kopfes zu achten.

Osteosynthesen. Wie bereits oben beschrieben, ist bei der Verwendung von Marknagelosteosynthesen auf den Eintrittspunkt und die Schraubenlage zu achten (- Abb. 5). Bei früheren, nichtwinkelstabilen Systemen gab es dabei häufiger Probleme mit der Schraubenverankerung im osteoporotischen Knochen [9]. Hinzu kommt die ebenfalls oben beschriebene Minimierung der Komplikationsrate durch das korrekte Besetzen sämtlicher Verriegelungsmöglichkeiten.
Bei der winkelstabilen Plattenosteosynthese treten ebenfalls versorgungsspezifische Komplikationen auf. Zum einen wurden bei älteren Plattensystemen durch den ausgeübten Druck häufiger sekundäre Nekrosen beobachtet, was jedoch bei neueren Plattendesigns deutlich rückläufig ist [6]. Zudem verfügen die winkelstabilen Platten auch bei osteoporotischem Knochen über ein stabiles Implantat-Knochen-Interface. Auch die vorhandene Elastizität des winkelstabilen Knochen-Implantat-Konstrukts erwies sich als günstig [15]. Dennoch sind auch bei den winkelstabilen Systemen der heutigen Generation weiterhin Schwierigkeiten vorhanden, wobei v. a. die Implantatauslockerung und der Materialbruch im Vordergrund stehen. Außerdem bereiten die unzureichenden Möglichkeiten der Tuberkelrefixation und sekundäre Oberarmkopfsinterungen Probleme [7]. Zudem ist bei der Osteosynthese darauf $\mathrm{zu}$ achten, dass die Platte nicht zu kranial positioniert wird, da es sonst in der Folge zu Impingementphänomenen kommt [15]. 


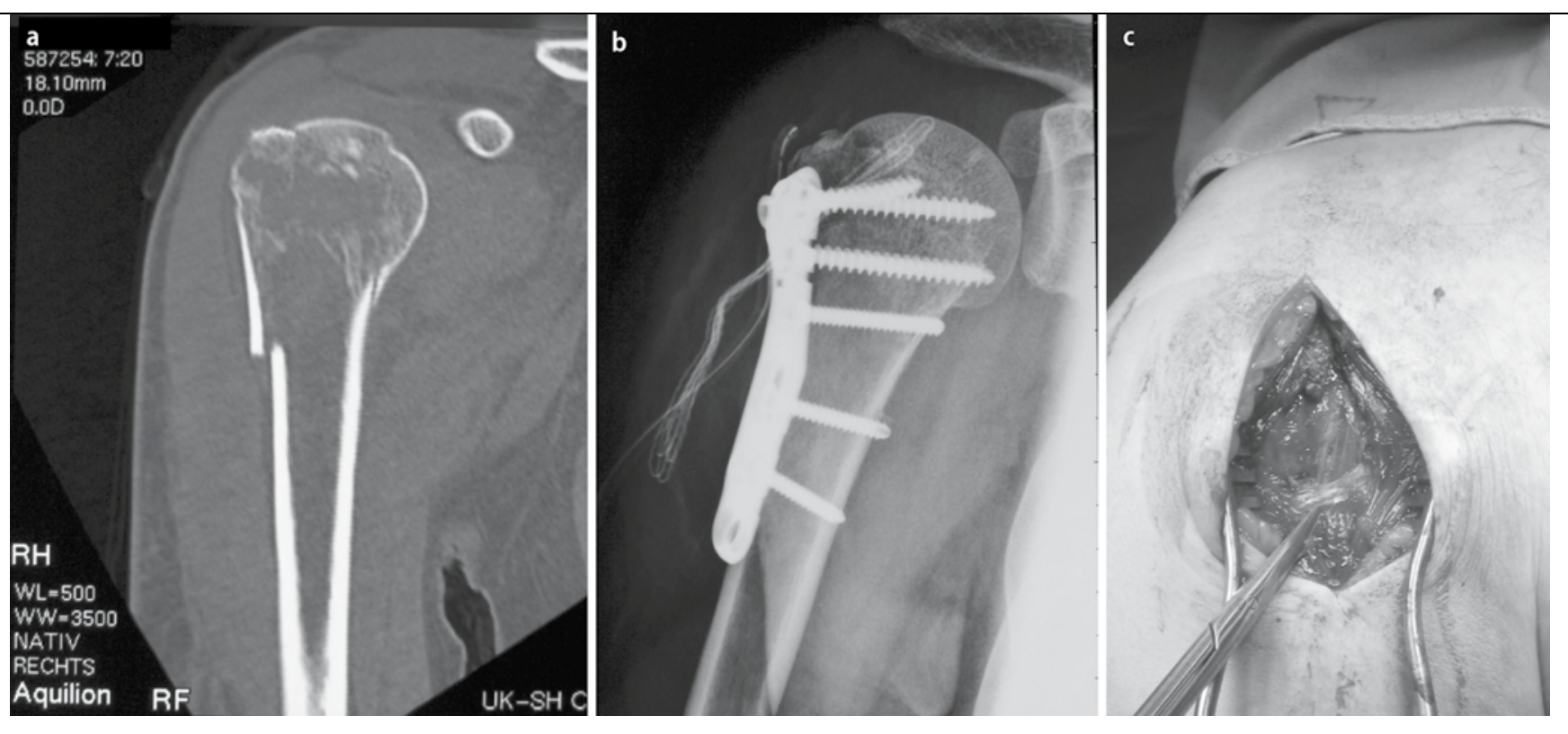

Abb. 4 \ Männlicher Patient, 37 Jahre, direktes Trauma mit subkapitaler Humerusfraktur (a), Versorgung über verlängerten

Deltasplitzugang mit Beachtung des Verlaufs des N. axillaris und eingeschobener Platte $(\mathbf{b}, \mathbf{c})$

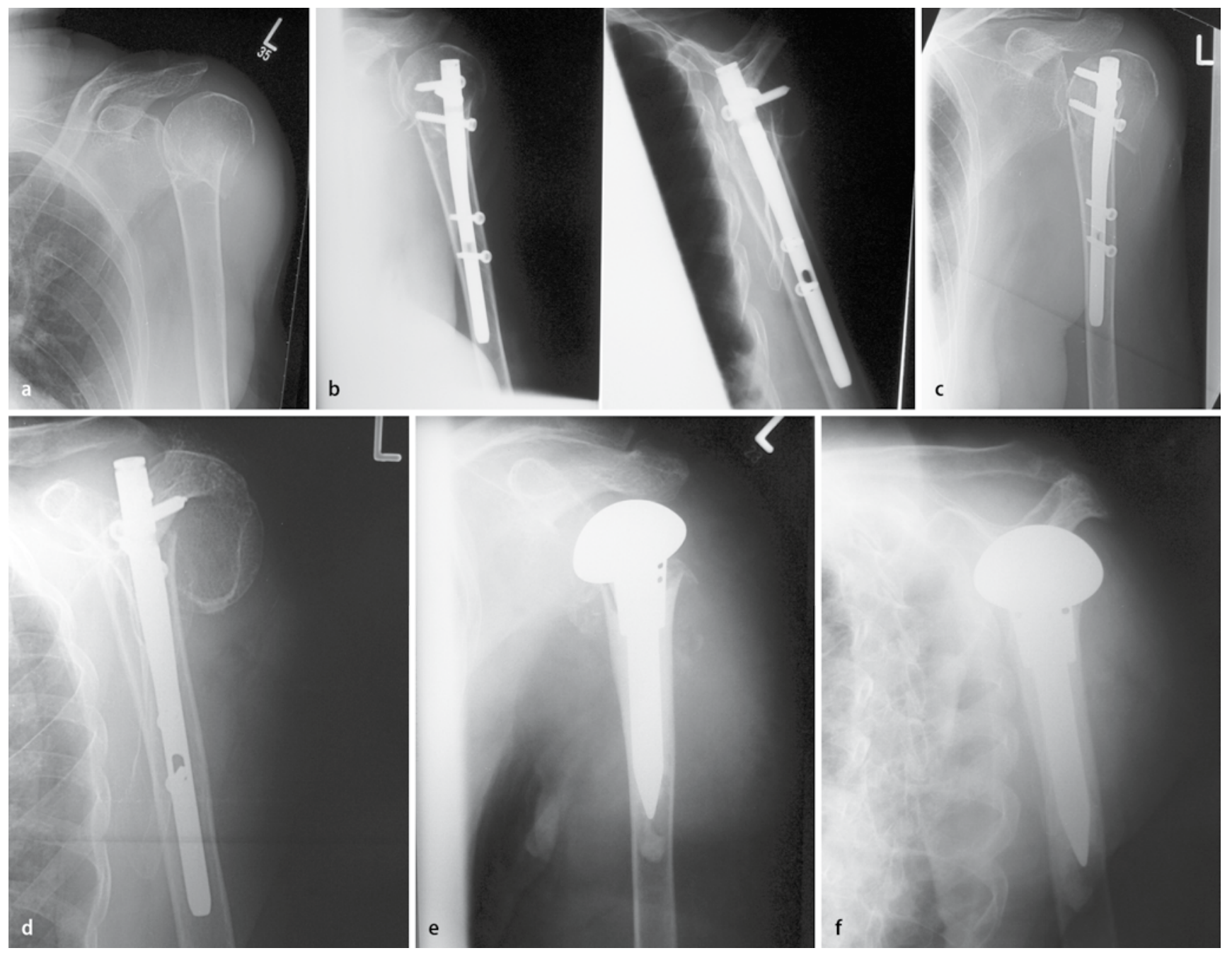

Abb. $5 \Delta$ Weibliche Patientin, 89 Jahre, direktes Trauma (a), initial Versorgung mit einem winkelstabilen T2-PHN (Fa. Stryker) $(\mathbf{b}, \mathbf{c})$, sekundäre Dislokation bei zu lateralem Nageleintrittspunkt und fehlender a.-p. Verriegelung im Humeruskopf (d), anschließende Versorgung mit Hemiprothese (e,f) 
Endoprothesen. Die primäre endoprothetische Versorgung gewinnt bei geriatrischen Patienten und v. a. bei komplexen Mehrfragment- und Head-Split-Frakturen weiter an Bedeutung [3].

Trotz aller funktionellen Defizite, die nach der endoprothetischen Versorgung auftreten - im Vordergrund stehen muskuläre Defizite durch mangelnde Tuberkelrefixation und ungenügende Rekonstruktion der Rotatorenmanschette $[1,4$, 16] - ist die primäre Versorgung v. a. bei der Behandlung geriatrischer Patienten unserer Meinung nach eine geeignete Option, da sie eine gute Schmerzreduktion bei oft zumindest befriedigender Funktion erreicht.

\section{Fazit}

Die Versorgung proximaler Humerusfrakturen stellt weiterhin ein umfangreiches Feld mit einer Vielzahl an Möglichkeiten dar. Es gilt, im klinischen Alltag immer wieder die Grundlagen zu beachten, um eine optimale Versorgung der Patienten zu gewährleisten. Dazu zählen einerseits die detaillierte Planung mittels präoperativer ausreichender Bildgebung und dem klinischen Bild, andererseits müssen auch individuelle Besonderheiten des Patienten selbst, wie v. a. der Compliance, und auch dessen Anforderungen an den Alltag in die Entscheidung mit einfließen. Nicht jede gleiche Fraktur gehört gleich versorgt.

\section{Korrespondenzadresse}

\section{Dr. A. Paech}

Sektion für Unfallchirurgie, Klinik für Chirurgie des Stütz- und Bewegungsapparates, Universitätsklinikum-Schleswig-Holstein, Campus Lübeck, Ratzeburger Allee 160, 23538 Lübeck Andreas.Paech@uk-sh.de

Interessenkonflikt. Der korrespondierende Autor gibt an, dass kein Interessenkonflikt besteht.

\section{Literatur}

1. Antuna SA, Sperling JW, Cofield RH (2008) Shoulder hemiarthroplasty for acute fractures of the proximal humerus: a minimum five-year followup. J Shoulder Elbow Surg 17: 202-209

2. Gerber C, Schneeberger AG, Vinh TS (1990) The arterial vascularization of the humeral head. An anatomical study. J Bone Joint Surg Am 72: 14861494
3. Gierer P, Simon C, Gradl G et al. (2006) Die Humeruskopfmehrfragmentfraktur - Versorgung mit einer Humeruskopfprothese? Orthopäde 35: 834840

4. Grönhagen $\mathrm{CM}$, Abbaszadegan $\mathrm{H}$, Révay $\mathrm{SA}$ et al. (2007) Medium-term results after primary hemiarthroplasty for comminute proximal humerus fractures: a study of 46 patients followed up for an average of 4.4 years. J Shoulder Elbow Surg 16: 766-773

5. Habermeyer P (Hrsg) (2002) Schulterchirurgie. Urban \& Fischer, München Jena

6. Hente R, Kampshoff J, Kinner B et al. (2004) Die Versorgung dislozierter 3- und 4-Fragmentfrakturen des proximalen Humerus mit einem winkelstabilen Plattenfixateur. Unfallchirurg 107: 769782

7. Hessler C, Schmucker U, Matthes G et al (2006) Ergebnisse nach Versorgung instabiler proximaler Humerusfrakturen mittels winkelstabiler Platte. Unfallchirurg 109: 867-874

8. Kollig E, Kutscha-Lissberg F, Roetman B et al. (2003) Primäre Hemiprothetik nach komplexem. Oberarmkopfbruch - funktionelle Spätergebnisse. Zentralbl Chir 128: 125-130

9. Maurer J, Rüter A-E (2004) Winkelstabile Plattenosteosynthese am proximalen Humerus. Ein klinischer Vergleich von etablierten und "neuen“ Verfahren. Trauma Berufskrankh [Suppl 2] 6: S247S250

10. Mittlmeier T, Stedtfeld H-W (2005) Stabilisation von Humeruskopffrakturen mittels antegrader winkelstabiler Verriegelungsmarknagelung (Targon PH). Trauma Berufskrankh [Suppl 1] 7:S15S26

11. Schippinger G, Szyszkowitz R, Seibert FJ (1997) Current concepts in the treatment of proximal humeral fractures. Curr Orthop 11: 203-214

12. Schmal H, Klemt C, Südkamp NP (2004) Stellenwert der Schulterprothese bei der Behandlung der 4-Fragment-Fraktur des Oberarmkopfes. Unfallchirurg 107: 575-582

13. Stedtfeld HW, Attmanspacher W, Thaler K et al. (2003) Fixation von Humeruskopffrakturen mit anterograder Marknagelung. Zentralbl Chir 128: 611

14. Voigt C, Lill H (2005) Winkelstabile Plattenosteosynthese proximaler Humerusfrakturen. Trauma Berufskrankh [Suppl 1] 7: S10-S14

15. Voigt C, Lill H (2007) Versorgung proximaler Humerusfrakturen. Trauma Berufskrankh [Suppl 1] 9: S43-S47

16. Ziegler J, Amlang M, Bottesi M et al. (2007) Ergebnisse endoprothetischer Versorgung bei Patienten vor dem 50. Lebensjahr. Orthopäde 36: 325-336 